Results Hyperoxia induced severe lung damage as evidenced by cell infiltration, edema and fibrosis which were reduced significantly by CDP-choline treatment. Radial alveolar count and lamellar body protein expression were significantly recovered, while number of TUNEL-positive cells and active Caspase- 3 expression were decreased by CDP-choline administration. Tissue proinflammatory cytokine (IL- $1 b$, IL- 6 and TNF- $\alpha$ ) levels as well as tissue MDA content and MPO activities were reduced, whereas GSH-Px and SOD activities were preserved in hyperoxia+CDP-choline group.

Conclusions Our data show for the first time that parenteral CDPcholine administration prevents hyperoxic lung injury in a neonatal rat model of BPD. It may therefore be suggested that CDP-choline can be used as an effective therapeutic agent for prevention of BPD in case it exhibits similar effects in humans.

\section{THE PRETERM PHENOTYPE - A COGNITIVE MODEL EXPLAINS THE PROSPECTIVE RELATIONSHIP BETWEEN PREMATURITY AND MATHEMATICAL PERFORMANCE DEFICITS}

doi:10.1136/archdischild-2012-302724.0129

1J Jaekel, ${ }^{2 D}$ Wolke. 'Developmental Psychology, Ruhr-University Bochum, Bochum, Germany; ${ }^{2}$ Psychology and HSRI, Warwick Medical School, University of Warwick, Coventry, UK

Background and aims The human brain is highly susceptible to the consequences of preterm birth. Cognitive tasks vary in complexity and resource requirements, thus performance on tasks with different demands may provide information on specific cognitive differences in children related to the degree of prematurity. Mathematical performance requires simultaneous processing of information which is particularly compromised in preterm children. Our aim was to investigate the relationships between task complexity and incremental performance deficits across the full spectrum of gestational age (GA).

Methods 1,513 children ranging from 27 to 43 weeks GA were studied from birth to 8 ; 5 years as part of a prospective geographically defined longitudinal investigation of neonatal at-risk children in South Germany (Bavarian Longitudinal Study). Children's cognitive performance at 8; 5 years was measured with $\mathrm{K}-\mathrm{ABC}$ subtests Number recall, Pattern reasoning, and Calculating and with a standardized mathematics test.

Results Results were twofold:

1. Preterm children showed incremental performance deficits with increasing task complexity.

2. There was a curvilinear relationship between GA and task performance with a point of change around 32 weeks of GA.

In general, every lost week of gestation increased the adverse impact on performance. However, this relationship was strongest among tasks which required the highest cognitive workload.

Conclusions With increasing cognitive workload preterm children fall behind in test scores. This suggests that brain organisation or damage limits cognitive resources. The relationship between GA and task performance is curvilinear. Our approach may offer a theoretical foundation to scrutinize the cognitive characteristics of the preterm phenotype.

\section{EXECUTIVE FUNCTION AND IT'S IMPACT ON MATHEMATICAL UNDERACHIEVEMENT AND ATTENTION PROBLEMS IN VERY PRETERM CHILDREN}

doi:10.1136/archdischild-2012-302724.0130

${ }^{1,2} \mathrm{CSH}$ Aarnoudse-Moens, ${ }^{2} \mathrm{~N}$ Weisglas-Kuperus, ${ }^{3} \mathrm{H}$ Duivenvoorden, ${ }^{1,4} \mathrm{JB}$ van Goudoever, ${ }^{5} \mathrm{~J}$ Oosterlaan. 1Pediatrics, Emma's Children Hospital, Academic Medical
Center, Amsterdam; 2Pediatrics, Erasmus MC - Sophia Childrens Hospital; ${ }^{3}$ Erasmus MC, Rotterdam; ${ }^{4}$ Pediatrics, VU University Medical Center; 5Child Neuropsychology, VU University, Amsterdam, The Netherlands

Background and aims Despite the dramatically increased survival rates for very preterm (gestational age $\leq 30$ weeks) infants, these children's developmental outcomes remain of significant concern. A majority of non-disabled very preterm children with IOs in the average range have substantial academic and behavior problems, of which deficits in mathematics and symptoms of inattention are the most pronounced. Executive function may be an important mechanism underlying these problems, an issue only scarcely examined and aim of this study.

Methods Two-hundred non-disabled very preterm (mean age $=$ $8.2 \pm 2.5$ ) and 230 term children (mean age $=8.3 \pm 2.3$ ), all born between 1996 and 2004, were assessed with measures of mathematics/arithmetic (Dutch Pupil Monitoring System), and executive function in preschool and in primary school. Parents and teachers reported on attention problems using the Achenbach behavior questionnaires and the Disruptive Behavior Disorders Rating scales.

Results Very preterm children had significantly more mathemati$\mathrm{cal}$ and attention problems than term children (SMD's $>0.46$ ). IO significantly predicted mathematical problems $(\beta \mathrm{s}>0.15, P \mathrm{~s}<0.04)$. Executive functioning, in particular spatial span and inhibitory control, was, over and above IO, significantly predictive for mathematical problems $(\beta s=0.11, P=0.003)$ and attention problems $(\beta s>0.17$ $P$ s $<0.001)$ in primary school. Associations were stronger for very preterm than for term children.

Conclusions Very preterm birth is associated with medium-sized deficits in mathematics and attention problems. Impaired executive function and IO scores are important predictors for these adverse outcomes.

\section{PHARMACOKINETICS AND CLINICAL EFFICACY OF PHENOBARBITAL IN ASPHYXIATED NEWBORNS TREATED WITH THERAPEUTIC HYPOTHERMIA}

doi:10.1136/archdischild-2012-302724.0131

'MPH van den Broek, ${ }^{2} \mathrm{~F}$ Groenendaal, ${ }^{2} \mathrm{MC}$ Toet, ${ }^{3} \mathrm{HLM}$ van Straaten, ${ }^{4} \mathrm{JGC}$ van Hasselt, ${ }^{4}$ ADR Huitema, ${ }^{2} \mathrm{LS}$ de Vries, ${ }^{1,5} \mathrm{ACG}$ Egberts, ${ }^{1} \mathrm{CMA}$ Rademaker. ${ }^{1}$ University Medical

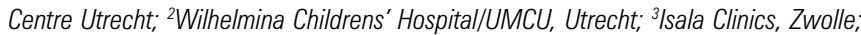
${ }^{4}$ Slotervaart Hospital, The Netherlands Cancer Institute, Amsterdam; ${ }^{5}$ Utrecht Institute for Pharmaceutical Sciences, Utrecht University, Utrecht, The Netherlands

Background and aims Therapeutic moderate hypothermia for neuroprotection in the asphyxiated newborn can influence pharmacokinetics and pharmacodynamics. If seizures occur, phenobarbital is the anticonvulsant of first choice. The aim of this study was to evaluate the effect of therapeutic hypothermia on phenobarbital pharmacokinetics and to evaluate the clinical efficacy of phenobarbital under hypothermia.

Methods Data were obtained from a prospective study in two Dutch level III NICUs (SHIVER-study). Term born newborns with criteria of perinatal asphyxia and encephalopathy were included. Therapeutic hypothermia $\left(33.5^{\circ} \mathrm{C}\right)$ was started within 6 hours after birth and was maintained for 72 hours. Pharmacokinetic modelling was performed using NONMEM.

Results In total, 31 term-born newborns were included of which 87 plasma samples were obtained (69 samples during the hypothermic phase). Based on a one-compartmental model with allometric relationships, clearance and distribution volume were estimated at $17.2 \mathrm{~mL} / \mathrm{h} / 3.5 \mathrm{~kg}$ and $3450 \mathrm{~mL} / 3.5 \mathrm{~kg}$ respectively. No relationship between hypothermia and pharmacokinetic parameters was identified. Overall, $66 \%$ of all neonates demonstrated sufficient seizure control with phenobarbital monotherapy, even though $69 \%$ of all measured concentrations were below $20 \mathrm{mg} / \mathrm{L}$. In $88 \%$ of neonates 